Classification

Physics Abstracts

$07.80-75.60-64.70 \mathrm{R}$

\title{
Use of in situ TEM experiments for phase transition studies
}

\author{
E. Snoeck $\left({ }^{1}\right)$, C. Roucau $\left({ }^{1}\right)$, P. Baules $\left({ }^{1}\right)$, M.J. Casanove $\left({ }^{1}\right)$, M. Fagot $\left({ }^{2}\right)$, B. Astie $\left({ }^{2}\right)$ and \\ J. Degauque $\left({ }^{2}\right)$
}

( $\left.{ }^{1}\right)$ CEMES-LOE/CNRS 29, rue Jeanne Marvig, F-31055 Toulouse, France

$\left({ }^{2}\right)$ Departement de Physique - INSA, avenue de Rangueil, F-31055 Toulouse, France

(Received January 20, 1993; accepted February 20, 1993)

\begin{abstract}
We present various in situ TEM experiments performed for phase transition studies. Such original experiments require original stages that are presented before each discussion. The first part concern TEM studies of ferroic domain switches carried out on ferromagnetic and ferroelectric/ferroelastic compounds. Then we present in situ TEM experiments carried out on the tetragonalorthorhombic structural phase transition in $\mathrm{YBa}_{2} \mathrm{Cu}_{3} \mathrm{O}_{6+x}$ under a controlled oxygen pressure. The last part of the paper deals with the incommensurate phase of Quartz. We present TEM experiments concerning the triple- $q$ to single- $q$ phase transition that have been performed using an heating straining stage and other original results evidencing the ferroelectric properties of the incommensurate phase.
\end{abstract}

\section{Introduction.}

TEM experiments have proved to be quite relevant for phase transition studies provide that the samples do not damage under electron irradiation. We present a review of original in situ TEM experiments performed on structural phase transitions, ferroic domain switches, and TEM studies of an incommensurate phase.

We first present the different kinds of ferroic compounds then we discuss results obtained using the 3 MV TEM of our laboratory on the ferromagnetic domain wall motions induced by the application of an external magnetic field for a Fe-Si alloy. We then expose TEM studies about the ferroelectric/ferroelastic domain switches observed with the application of an external electric field on polycrystalline $\mathrm{BaTiO}_{3}$ based ceramics.

The second part of the paper deals with structural phase transitions associated to variations of stoichiometry. Such experiments could be performed at different temperatures using an environmental cell available on the 3 MV TEM of our laboratory. We illustrate such in situ experiments by results obtained on the tetragonal to orthorhombic phase transition in $\mathrm{YBa}_{2} \mathrm{Cu}_{3} \mathrm{O}_{6+x}$ compound studied under oxygen pressure.

In the last part, we discuss TEM results obtained at high temperature on the incommensurate phase of Quartz under stress and with the application of an external electric field. 


\section{Studies of ferroic domain wall motions.}

2.1 InTRODUCTION. - A ferroic crystal is a compound that contains two or more stable domains which can be switched by a suitably external driving force [1]. We may define primary ferroic class for which these domains differ in spontaneous values of the magnetization (for ferromagnetism), polarization (for ferroelectric compounds) or stress (for ferroelastic materials). The switches of these orientation states may be accomplished by magnetic field, electric field and mechanical stress respectively. These switches occur by the motion of the domain walls that stabilizes a large single domain displaying the more stable orientation state compared to the direction of the driving force. Friction forces, interaction with local defects, dislocations, grain boundaries etc... could pin the boundary motions. They are responsible of the hysteresis loop shape of the macroscopic parameter variation (magnetization, polarization, stress) as a function of the external field (magnetic, electric strain). It corresponds to the energy losses associated to the switches. The knowledge of the different microscopic phenomena that occur during the domain wall motion is then important to understand and minimize these energy losses.

In order to study by TEM the ferroic domain wall motions and their interactions with structural defects for each ferroic compounds, we have realized various stages permitting the application of external magnetic field, external electric field or external stress at various temperatures.

We present the original stages and results obtained on the ferromagnetic domain wall motions in Fe-Si alloy and on the ferroelectric/ferroelastic domain boundary switches in $\mathrm{BaTiO}_{3}$.

\subsection{BLOCH WALL MOTION IN Fe-Si ALLOY.}

2.2.1 Experimental. - The observations of magnetic domain walls (Dw's) by TEM in Lorentz's mode, require to set the samples in a region of the objective lens where the magnetic field has a low value. This is achieved on the HVEM $3 \mathrm{MV}$, using new pole-pieces which rise the specimen, so as to decrease the axial field on the foil from 25000 Oe down to 700 Oe. The long focal length induces a decrease of the resolution that it is improved by the low value of $\lambda$ at $1 \mathrm{MV}$ and is around $2 \mathrm{~nm}$. The stage for the Dw's dynamic observations is presented in figure 1. Two symmetrical coils (with 60 turns of copper wire) allow magnetic fields to be applied. The samples are glued to the ends of the cores, using a high permeability and conductibility glue. According to the nature of the grips (soft ferromagnetique "alloy-hyperm0" or copper) the beam deflection is negligible. The applied magnetic field " $H_{\mathrm{a}}$ " values have been computed using a program for computing the field in electronic lenses. The experimental values have been measured with a Hall effect gauge and fit quite well with the computed ones. They vary between 0 and 50 Oe. Using this device, Dw's can be imaged in Fresnel's mode and magnetic domains in Foucault's mode of observation.

For the measurement of the hysteresis loops, the variations of $\mathrm{H}_{\mathrm{a}}$ are monitored manually, allowing the Dw's to jump from one equilibrium position to another one, as done by Taylor [2]. The recorded motions of the Dw's give the variations of the magnetization of the adjacent magnetic domains.

2.2.2 Results. - We have studied the interaction between Dw's and various structural defects: grain boundaries and antiphase boundaries (APB's) in a Fe-Si crystalline alloy with $6.5 \%$ wt Si. $\mathrm{Fe}-\mathrm{Si}$ alloys are very important for electrotechnical applications. The most interesting magnetic features (for instance the lowest losses) correspond to the amount of $6.5 \% \mathrm{wt} \mathrm{Si}$. According to the brittleness of the Fe-Si alloy at this composition, we have got it using fast solidification by planar flow casting method. After quenching, the ribbons are recrystallized for $2 \mathrm{~h}$ at $1050{ }^{\circ} \mathrm{C}$. The average grain size is $300 \mu \mathrm{m}$ and the density of dislocations about $10^{-6} \mathrm{~cm}^{-2}$. After solidification some B2 type ordering appears [3]. After annealing at $1050{ }^{\circ} \mathrm{C}$ the average size of the 


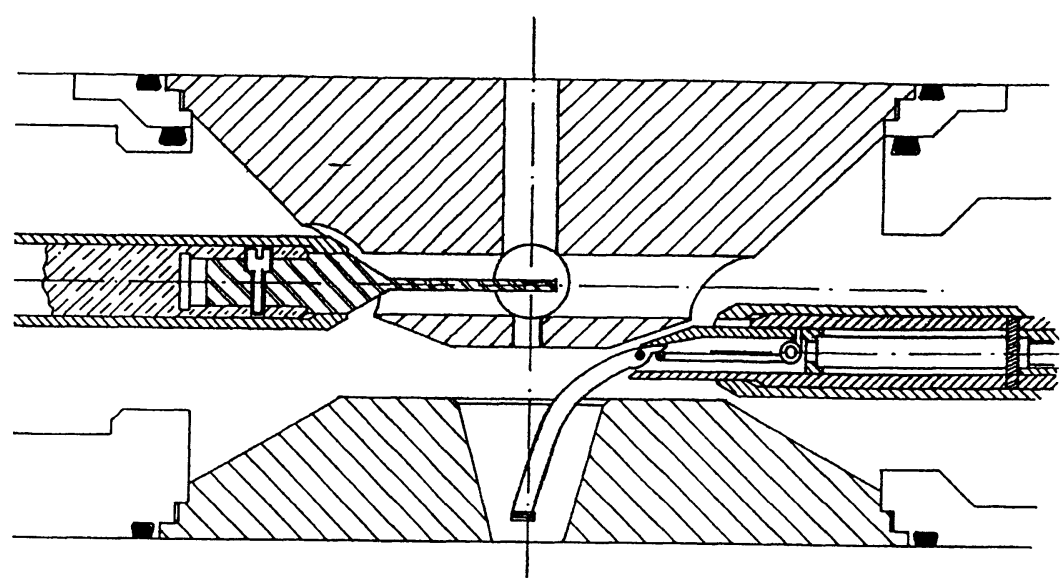

Fig. 1. - Diagram of the experimental setting permitting the in situ application of a magnetic field.

ordered domains increases from 15 to $125 \mathrm{~nm}$. In order to improve the magnetic properties and to perform more accurate study of the interaction between Dw's and APB's, the samples have been reannealed for $2 \mathrm{~h}$ at $700{ }^{\circ} \mathrm{C}$. This induces an additional increases of the ordered domain size which reaches $3.5 \mu \mathrm{m}$ in average.

2.2.2.1 Interaction between Dw's and grain boundaries. - An hysteresis loop in bright field mode of Lorentz's microscopy has been studied on a sample annealed at $1050{ }^{\circ} \mathrm{C}$. In figure $2 \mathrm{a}$, different positions of the magnetic configuration observed in Fresnel's mode are presented for various values of the applied field $H_{\mathrm{a}}$. In order to increase the magnetic domains contrasts some micrographs have been taken in Foucault's mode. The scheme of the Dw's interactions with a grain boundary between two grains tilted by an angle of $15^{\circ}$, is schematized figure $2 \mathrm{~b}$. The directions of $H_{\mathrm{a}}$ and of the projections of the $\langle 100\rangle$ set of the saturation magnetizations " $M_{\mathrm{s}}$ " are reported. Two hysteresis loops have been measured: one corresponds to the motion L2 of the segment PQR of a $90^{\circ} \mathrm{Dw}$ (Fig. 3a), the other to the displacement L1 of the segment RS of a $180^{\circ} \mathrm{Dw}$ (Fig. 3b). The run of $H_{\mathrm{a}}$ during the cycles is reported in figure 2c. The $90^{\circ} \mathrm{Dw}$ interacts directly with the grain boundary. We have also studied the behaviour of the area "a" of the closure domain built on the grain boundary (Fig. 3c). The two loops show similar features presenting a non symmetrical shape, a low remanent magnetization and quite similar values of the local thin foil coercivity " $h_{\mathrm{c}}$ " $(\sim 1.7 \mathrm{Oe})$. For the negative branch $\left(H_{\mathrm{a}}\right.$ decreasing from 0 to $\left.-2.8 \mathrm{Oe}\right)$ inverse jumps can be observed corresponding to the interaction with other Dw's.

The higher hysteresis losses due to the $90^{\circ} \mathrm{Dw}$ (the average values of the increments of $\mathrm{L} 1$ and L2 are respectively $0.139 \mu \mathrm{m}$ and $0.487 \mu \mathrm{m}$ ) can be explained considering that the direction of $H_{\mathrm{a}}$ is more favorable (1.4 time more important) for the motion of the PQR magnetic wall than for the RS one. So the direction of the motion of the $90^{\circ} \mathrm{Dw}$ is roughly parallel to the grain boundary, giving quite large jumps. The $180^{\circ} \mathrm{Dw}$ does not only move in a parallel direction with the grain boundary but also perpendicularly to it, it then interacts much more with the smooth force fields of the defects inside the grains. The variations of "a" during the loop which are more similar to those of L2 than of L1 confirm our hypothesis (cf Figs. 3a and 3c).

2.2.2.2 Interaction between Dw's and APB's. - The analyses of the interactions between Dw's and defects inside the grains, have been performed in dark field mode with $a(001)$ superlattice reflection, on a sample annealed for $2 \mathrm{~h}$ at $700{ }^{\circ} \mathrm{C}$. Several equilibrium positions for different 

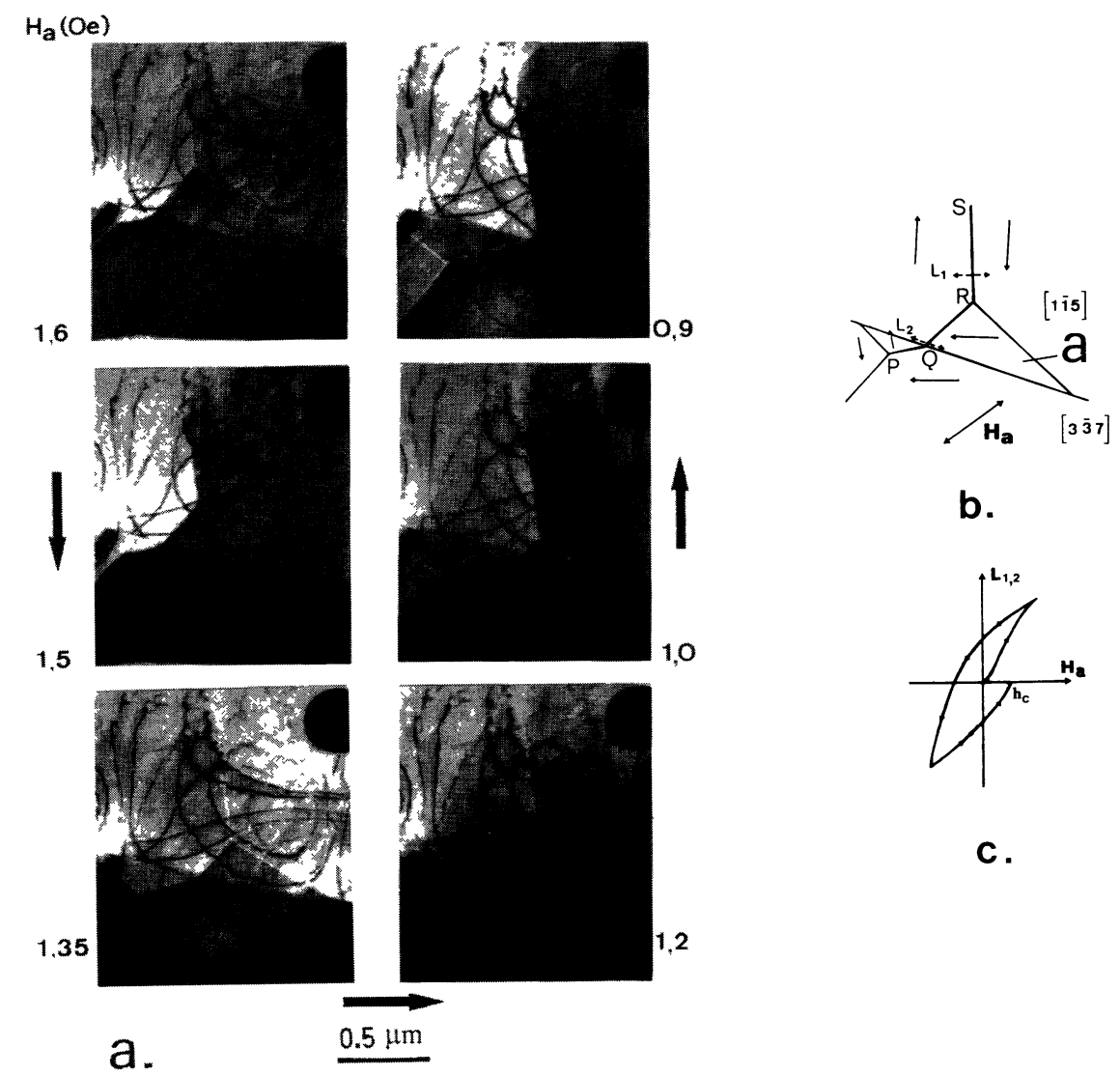

b.

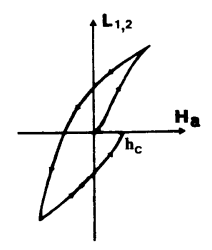

c.

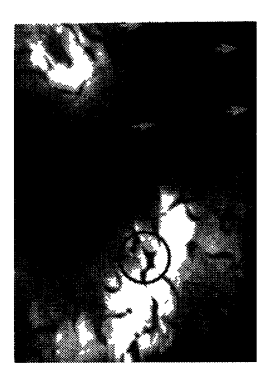

$2.5 \mathrm{Oe}$

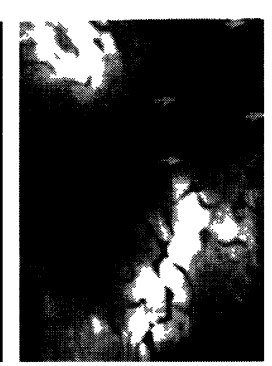

$2.1 \mathrm{Oe}$

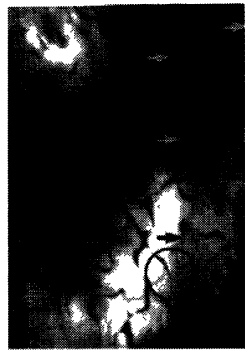

$1.7 \mathrm{Oe}$

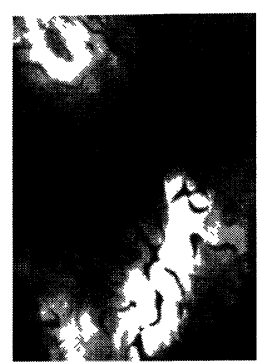

$0 \mathrm{Oe}$

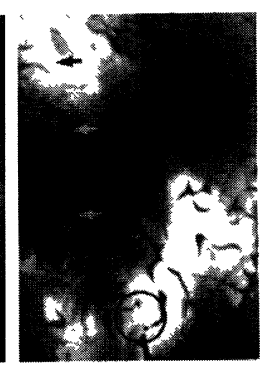

$-0.4 \mathrm{Oe}$

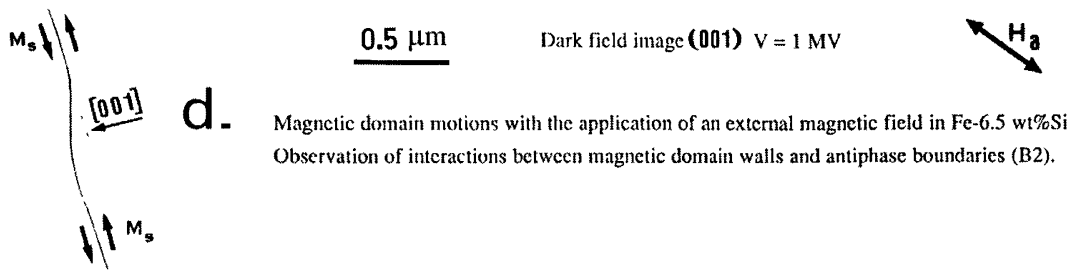

Fig. 2. - Interaction between DW's and structural defects. a): grains boundaries, b): scheme of the distribution of $M_{\mathrm{s}}, \mathrm{c}$ ): scheme of the $H_{\mathrm{a}}$ run in the hysteresis loop, d) Antiphase boundaries (APB's). 

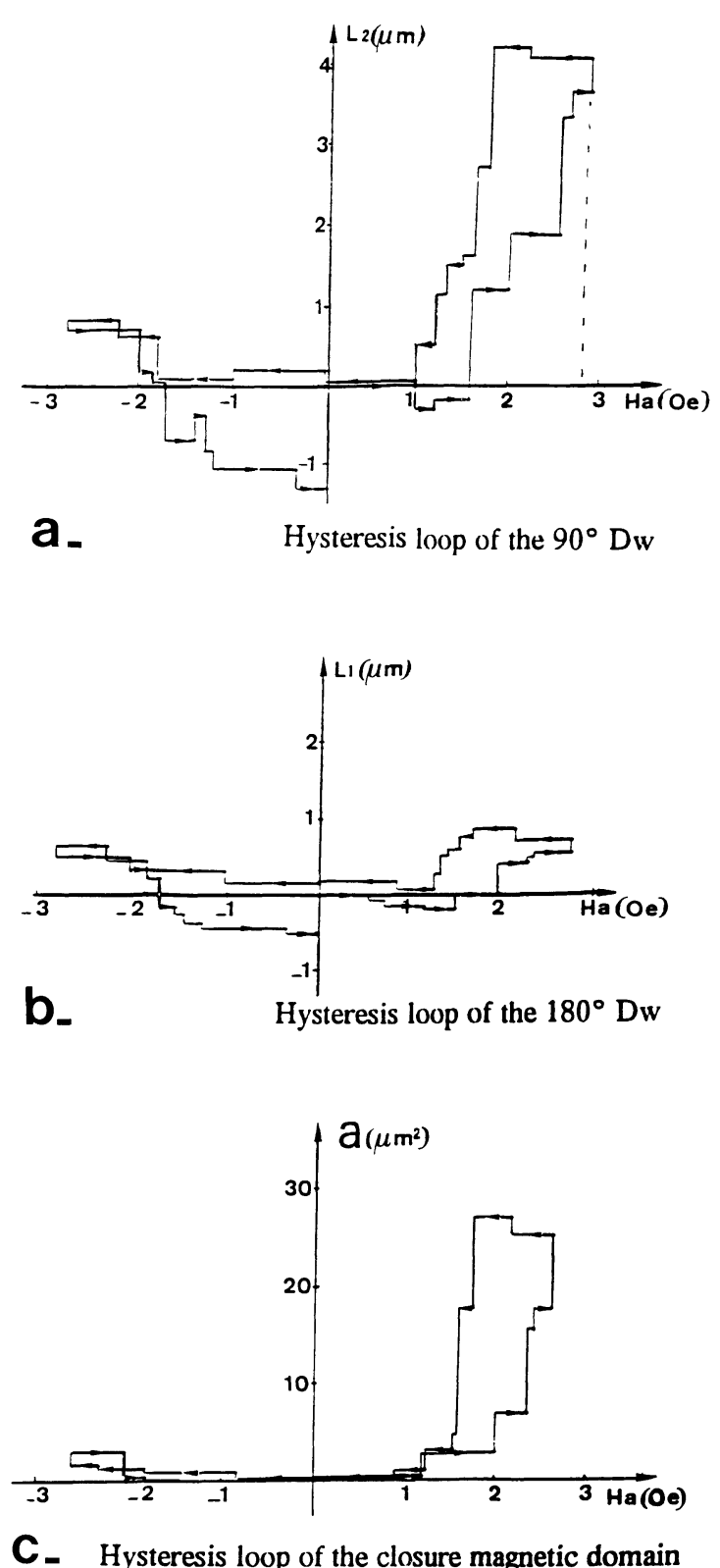

Fig. 3. - Hysteresis loop of a): $90^{\circ} \mathrm{DW}$ (PQR segment), b): $180^{\circ} \mathrm{DW}$ (RS segment), c): area " $a$ " of the closure domain.

values of $H_{\mathrm{a}}$ of a "white" $180^{\circ} \mathrm{Dw}$ inside a surstructure of the B2 type, are presented in figure $2 \mathrm{~d}$. As circled on the bottom of the micrograph, a pinning effect between the Dw and the planar (001) 
part of the APB's appears. Our TEM results give then an evidence of the interaction between Dw's and APB's in the Fe-Si $6.5 \%$ wt alloy. Furthermore our measurements of macroscopic magnetic hysteresis loops and our computations show that the interaction field of forces between Dw's and APB's in such alloys is very small that is in good agreement with our observations of hysteresis loops in Lorentz's microscopy [4].

2.2.3 Conclusions. - The in-situ TEM experiments in Lorentz's mode are very useful for studying ferromagnetic compounds which exhibits several types of structural defects. It allows to distinguish the different mechanisms of interaction with the Dw's and to precise their respective contribution to the magnetic hysteresis [4]. It also permits to study the pinning anisotropy of the Dw's by grain boundaries with more precision than by macroscopic measurements. The TEM Lorentz's microscopy presents also the advantage, due to the peculiarities of the $3 \mathrm{MV}$ microscope, to study the behaviour of the Dw's under various fields (stress or magnetic fields) applied separately or together.

\subsection{FERROELECTRIC/ FERROELASTIC DOMAIN WALL MOTIONS IN $\mathrm{BaTiO}_{3}$.}

2.3.1 Experimental. - A commercial Philips $\pm 40^{\circ}$ heating stage has been transformed. In this experimental setting the sample is sandwiched between two copper apertures realizing the electrodes as it is presented on figure 4 . The samples are $3 \mathrm{~mm}$ diameter discs that have been mechanical polished until a $300 \mu \mathrm{m}$ thickness has been reached. The final thinning for the electron transparency was achieved by argon ion milling. The whole "sandwiches" (the sample and the electrodes) is glued using an insulator ceramic paste. The thermocouple threads of the original heating sample holder have been replaced: one is soldered on the crucible and the other one on the upper copper aperture. This allows the polarization of the sample through the two copper electrodes. The sample could be heating by tailoring the current intensity in a thermal resistor. The effects of the electric field have to be observed near the edge of the central hole of the electrodes where the electric field presents a small curvature (see Fig. 4).

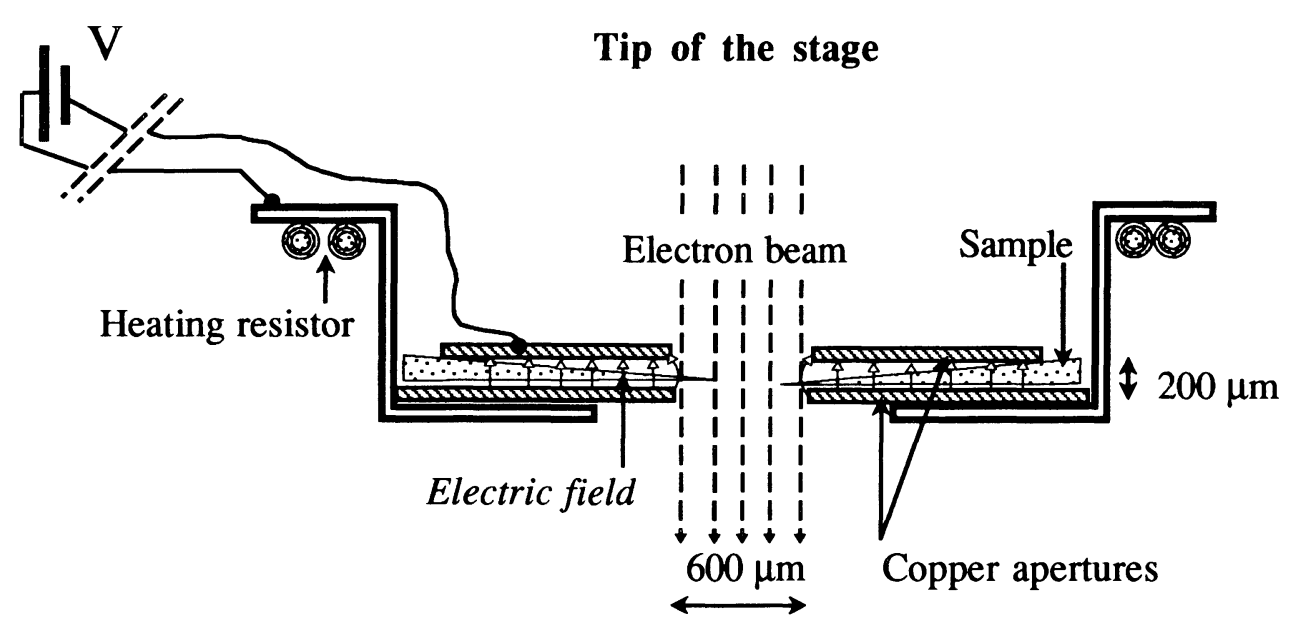

Fig. 4. - Diagram of the experimental setting permitting the in situ application of an electric field. 
2.3.2 $\mathrm{BaTiO}_{3}$. - - Barium titanate exhibits a perovskite cubic structure from $130{ }^{\circ} \mathrm{C}$ up to $1460^{\circ} \mathrm{C}$. At $130^{\circ} \mathrm{C}$ a phase transformation occurs and the cubic cell of the perovskite form elongates along an edge to a tetragonal non-centrosymmetric structure. The tetragonal phase is ferroelectric and partially ferroelastic. It displays 6 ferroelectric states (with the polarization vector parallel or antiparallel to the " $a$ " axis of the cubic cell) and 3 ferroelastic domains. Considering the orientation of the polarization, the ferroelectric domain walls of the $\mathrm{BaTiO}_{3}$ tetragonal phase could be described as $90^{\circ}$ domain boundaries which separate ferroelastic domains (that have their polarization at $90^{\circ}$ one to the other) and $180^{\circ}$ domain walls which separate antiparallel domains (these domains belong to a same ferroelastic state). The first walls belong to the $\{110\}$ family and the pure ferroelectric domain boundaries belong to the $\{001\}$ one.

2.3.3 TEM observation of domain wall motions induced by an electric field. - We have reported in figures 5a-c bright field micrographs of the same area observed for different values of the electric field: from $12 \mathrm{kV} / \mathrm{cm}$ to $24 \mathrm{kV} / \mathrm{cm}$. On the micrograph figure 5 a two kinds of $90^{\circ}$ walls can be observed. They are [110] and [-110] planes. When the electric field is increased the domain structure changes and a large monodomain takes place as it can be seen on the top right of the micrograph in figure $5 \mathrm{~b}$. The growth of this new state has been obtained by the motion of the domains tips as it as already been observed by Bornarel et al. for KTP compound by optical method [5]. We have pointed out with arrows in figure 5a the displacements of domain points that create the new state. This motion is energetically easier compared to the lateral motion of walls because it just involves displacements of the atoms located near the domain point. This displacement occurs until the point cross the whole sample as it is observed in figure 5c. The motion velocities have been measured from the video record with an order of magnitude close to $\approx 5 \mu \mathrm{m} / \mathrm{sec}$.

When the domains cross the whole sample or when the tip is pinned, the increase of the domain

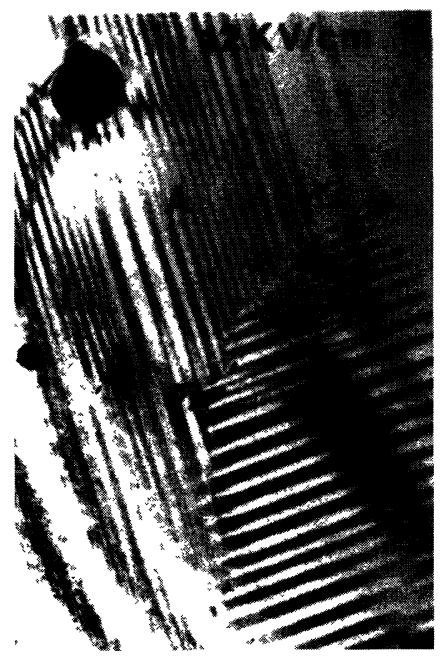

a)

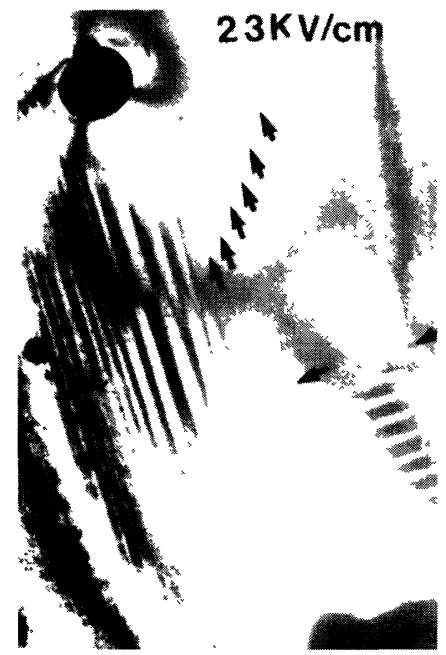

b)

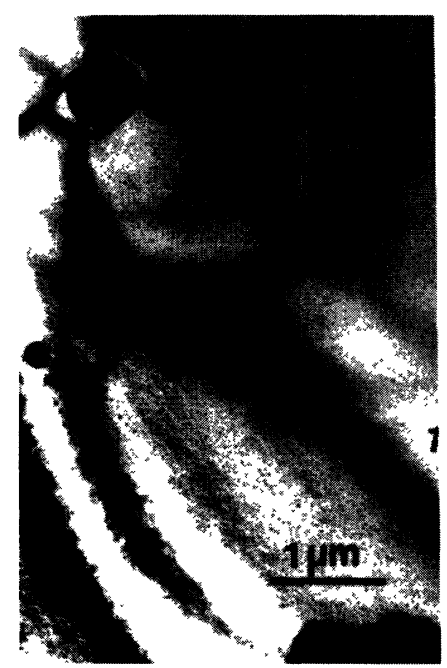

c)

Fig. 5 a-c. - Ferroelectric/ferroelastic domain wall motions in $\mathrm{BaTiO}_{3}$. Bright field images obtained on a same area for different values of the electric field. a) $12 \mathrm{kV} / \mathrm{cm}$, b) $23 \mathrm{kV} / \mathrm{cm}$, c) $24 \mathrm{kV} / \mathrm{cm}$. 
size could only be obtained by a lateral motion of walls. Such observations have been reported figure 6a-c. They are 200 dark field images of a same area observed at room temperature. The voltage has been increased from 0 to $2 \mathrm{kV} / \mathrm{cm}$ then reversed to $-2 \mathrm{kV} / \mathrm{cm}$. The bottom right of the micrographs corresponds to the edge of the sample. We have arrowed a lateral motion of the wall (Figs. 6a to b). As discussed above this motion stabilizes the most energetically favorable state and so there is no reason for this motion to stop. Nevertheless this lateral displacement involves a coherent shift of all the atoms located along the boundary. We then assume that only some defects set along this wall should be able to lock the motion of the whole boundary as it is observed on the figure $6 \mathrm{~b}[6]$. The micrograph figure $6 \mathrm{c}$ has been obtained after having increasing the electric field until the stabilization of one monodomain then decreasing down to $-2 \mathrm{KV} / \mathrm{cm}$. A regular arrangement of ferroelectric domains $0.2 \mu \mathrm{m}$ in size is observed and should be more stable since it still occurs'for the same absolute value of the electric field than in the situation in figure 6 .

\section{TEM studies of phase transitions under gas pressure.}

3.1 EXPERIMENTAL. - An environmental cell available on a $3 \mathrm{MV}$ TEM has been realized in our laboratory [7]. It allows to perform TEM experiments under gas pressure varying from 0 to $\approx 450$ Torr and at various temperatures using heating or cooling stages. Two $100 \mu \mathrm{m}$ diameter apertures permit to isolate a room in which the pressure can be controlled up to 400 Torr. Two other $200 \mu \mathrm{m}$ diameter apertures separate an intermediate cell from the vacuum of the column (about $10^{-6}$ Torr) and the previous room. The specimen holder used for the experiments is a heating stage on which the temperature is measured by a thermocouple chromel-alumel.

3.2 $\mathrm{YBa}_{2} \mathrm{Cu}_{3} \mathrm{O}_{6+x}$. - As it is now well known, the $\mathrm{YBa}_{2} \mathrm{Cu}_{3} \mathrm{O}_{6+\mathrm{x}}$ type compounds crystallize in the orthorhombic or in the tetragonal system depending on the value of the oxygen content i.e. when $x$ is close to 0 the compound is in the tetragonal phase while the orthorhombic phase is obtained for $x$ close to 1 . A compound in the orthorhombic phase undergoes a structural phase transition and becomes tetragonal when it is heated above a certain temperature. If the compound is cooled down under an oxygen flow, the sample increases its oxygen content and crystallizes again in the orthorhombic phase. This reversible structural transition has already been studied by $\mathrm{X}$ ray or electron diffraction $[8,9]$. Depending on the material, the temperature of the phase transition lies around $600-700{ }^{\circ} \mathrm{C}$. When looking at the structure of these compounds the phase transition results from the loss of the oxygen atoms lying in the $\mathrm{CuO}$ planes. It has been shown that the presence of these atoms is essential for the occurrence of a super-normal transition. In these compounds, the superconductivity appears under $92 \mathrm{~K}$ for $x \geq 0.62$ i.e. in the orthorhombic phase.

The structural phase transition has been studied on a compound, ( $\mathrm{Ln}) \mathrm{Ba}_{2} \mathrm{Cu}_{3} \mathrm{Cu}_{3} \mathrm{O}_{6+x}$, which is isostructural with $\mathrm{YBa}_{2} \mathrm{Cu}_{3} \mathrm{O}_{6+x}$, but presents better morphological characteristics as bigger grains with cleam grain boundaries and a narrow superconducting transition [10].

The orthorhombic phase is ferroelastic and then characterized by the presence of domains due to multiple twinning that occurs during the tetragonal-orthorhombic transition. The presence or the absence of twins, easily recognizable in electron microscopy image mode, gives therefore a useful indication on the nature of the observed phase (orthorhombic or tetragonal respectively) $[9,11]$. 

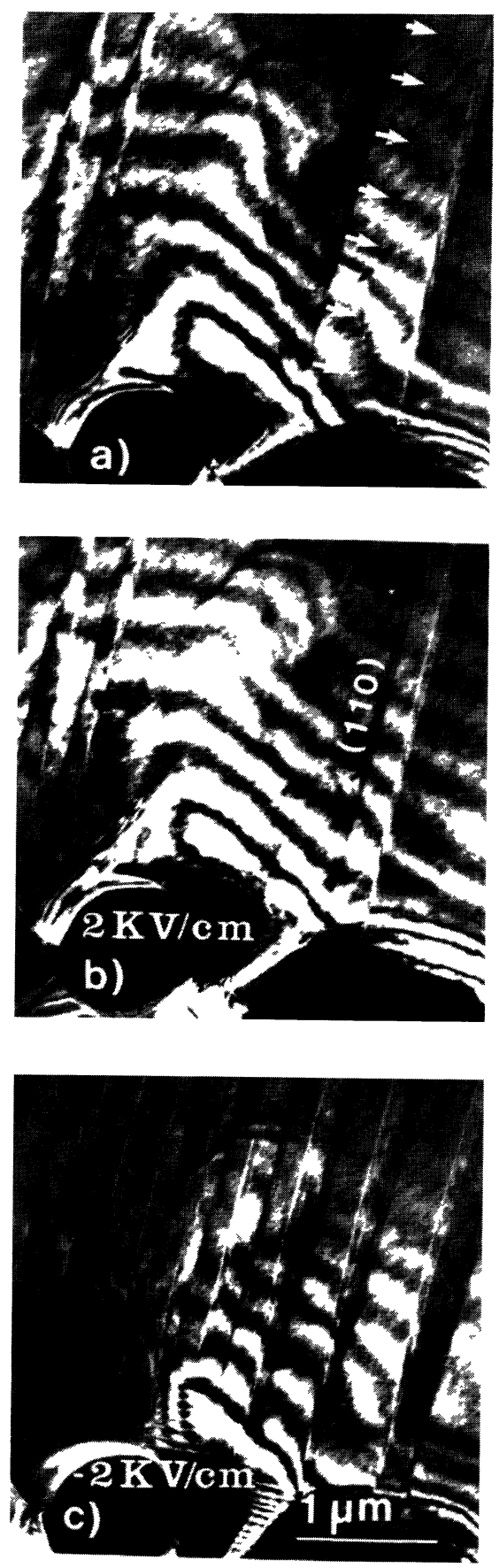

Fig. 6 a-c. - (200) dark field micrographs obtained for different values of the electric field. a) $0 \mathrm{kV} / \mathrm{cm}, \mathrm{b}$ ) $2 \mathrm{kV} / \mathrm{cm}, \mathrm{c})-2 \mathrm{kV} / \mathrm{cm}$. 


\subsection{RESULTS.}

3.3.1 Phase transition temperature versus oxygen pressure. - The sample which starting composition is close to the stoichiometry "O7", is fixed on the heating stage and inserted in the environmental cell under a selected oxygen pressure. The temperature of the sample is then increased until the twins disappear. These experiments have been reproduced at the successive oxygen pressures 45, 50, 70, 80 and 100 torrs. The results are reported on the graph in figure 7 . The general aspect of the graph suggests that above a certain oxygen pressure, the transition temperature remains constant.

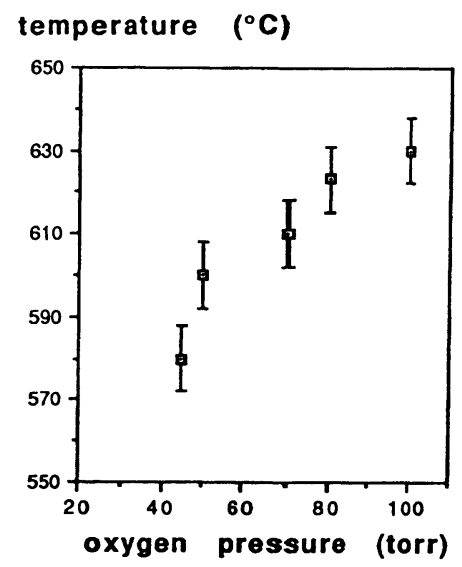

Fig. 7. - Structural phase transition in $\mathrm{YBa}_{2} \mathrm{Cu}_{3} \mathrm{O}_{6+x}:$ Graph of the phase transition temperature versus oxygen pressure.

3.3.2 Kinetics of the oxygen desorption. - In this experiment, the sample is first annealed under an oxygen flow in order to stabilize the orthorhombic phase. Then the sample lying under primary vacuum, is heated rapidly to a chosen temperature. We measure the time for the twins to disappear that is therefore the time required for the phase transition to occur, the transition being only due to the oxygen desorption. This experiment has been performed at several temperatures from two kinds of initial conditions. The results are presented in figure 8.

3.3.3 Kinetics of the oxygen absorption. - Starting in the tetragonal phase stabilized at fixed conditions (in temperature and time) the temperature is reduced to a chosen value and oxygen is introduced at a selected pressure in the environmental cell. We then measure the time for the twins to appear. This experiment has been performed at different temperatures for a given $\mathrm{P}_{\mathrm{O} 2}$ with the same initial conditions, the whole process being repeated for different pressures.

The analysis of the results so obtained leads us to distinguish between two different regions. Under a given temperature, $T_{0}$ (approximately $560^{\circ} \mathrm{C}$ ), the phase transition occurs sooner with increasing temperatures. Above $T_{0}$, the time required for the transition to occur increases until a quasi-infinite value. This last behaviour indicates the approach of the temperature domain where the tetragonal phase is the most stable one. The shortest time observed in all the graphs in figure 9, indicates the existence of an optimum temperature of annealing under oxygen in these compounds. 

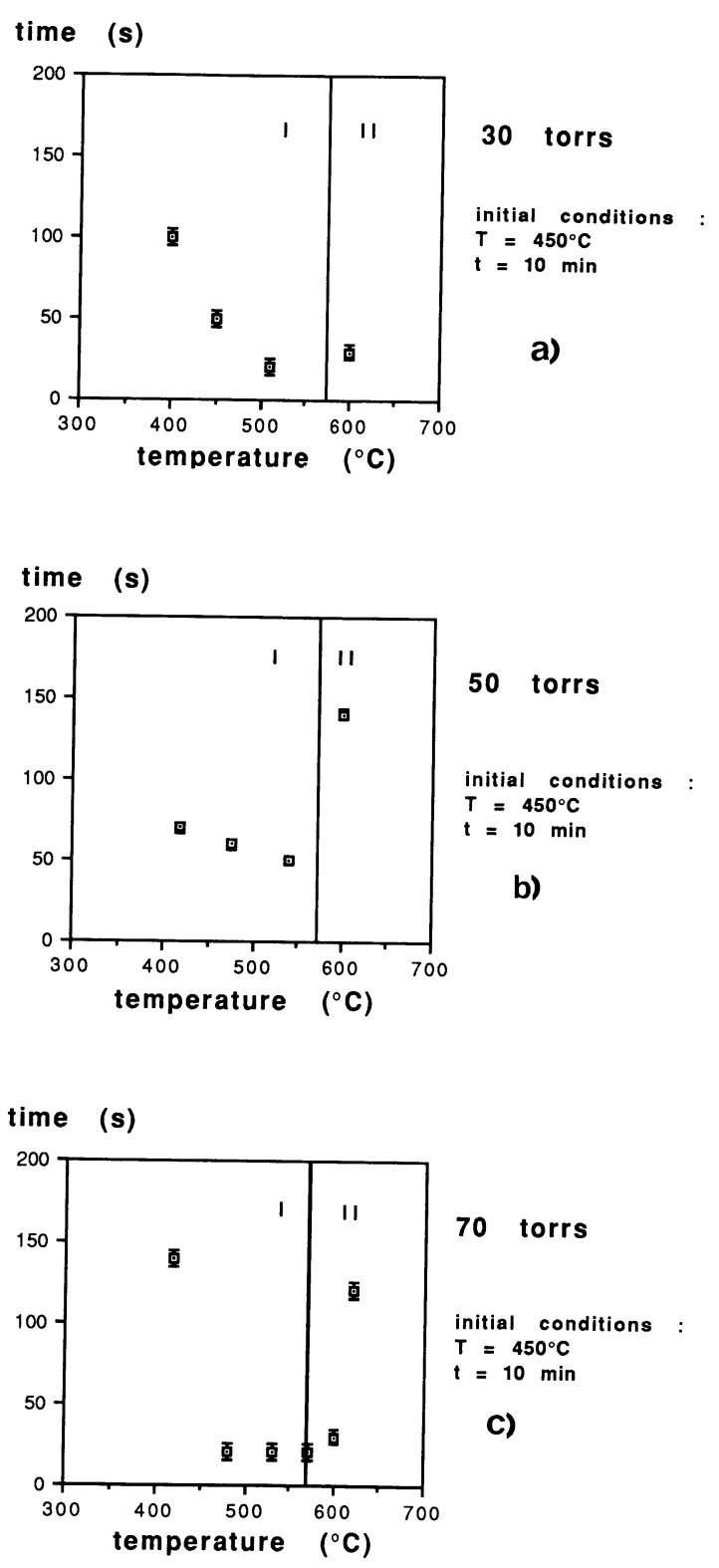

Fig. 8. - Kinetics of the phase transition as a function of the temperature under the selected oxygen pressures : a) 30 Torrs ; b) 50 Torrs ; c) 70 Torrs. 


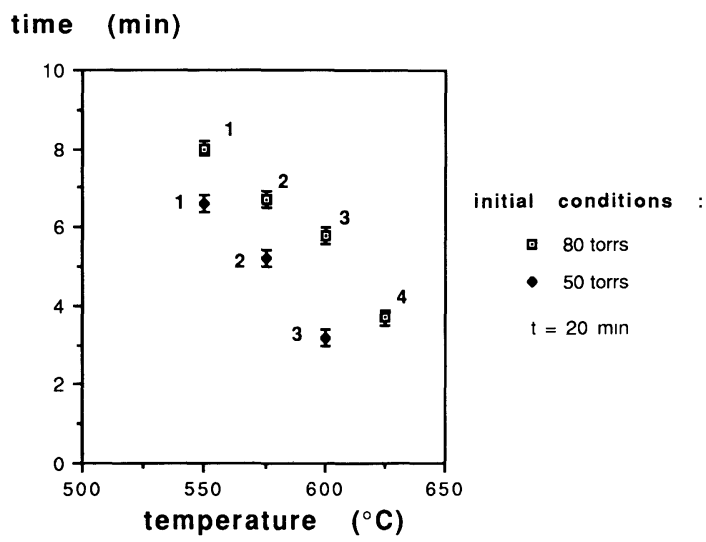

Fig. 9. - Kinetics of the phase transition as a function of the temperature under primary vacuum. Initial annealing conditions: $20 \mathrm{mn}$ under 50 and 80 Torrs of oxygen

3.4 DisCUSSION. - Those results have already been discussed in [11]. We only report here the main results. Chemisorption at the surface, diffusion in the lattice and fixation on particular sites $\left(\mathrm{O}_{(4)}\right)$ are the different mechanisms involved in the oxygen absorption and desorption phenomena. Depending on the thermodynamical conditions, one of these mechanisms can prevail over the others. The aspect of the graphs figure 8 and in region I of figure 9 shows that the determining mechanism is thermically activated. It can therefore be thought that the diffusion of oxygen in the lattice governs the kinetics of oxygen desorption and oxygen absorption in the region I of figure 9. In region II the chemisorption can be restrained at high temperatures so that the capture of oxygen by the lattice becomes more difficult i.e. the thermal excitation may prevent oxygen atoms from fixing on a preferential site. At higher temperatures the time required for the transition to occur becomes infinite since only the tetragonal phase is stable.

\section{TEM studies of the incommensurate phase of quartz.}

4.1 InTRODUCTION. - Quartz displays an incommensurate phase at the $\alpha$ - $\beta$ phase transition around $850 \mathrm{~K}$ [12-17] that has been successfully explained by Aslanyan and Levanyuk [18] using the Landau theory of phase transition. This modulated phase is a triple- $q$ modulation, the modulation star is composed of three equal length vectors at $120^{\circ}$ from each other and tilted by an angle $\pm \varphi$ from the $y$-directions as evidenced by different experiments leaded in the real space [12-14] and in the reciprocal one [15-17]. The two equivalent values $+\varphi$ and $-\varphi$ of wave vector star angle induce the presence of " $\pm \varphi$ macrodomains". Recently Dolino et al. [19] have shown that the an uniaxial stress induces a phase transition in the incommensurate phase of Quartz from a triple- $q$ modulated phase to a single- $q$ one. Theoretical investigations of Vallade and Walker have suggested that those blocks should exhibit antiparallel polarization along the $c$ axis [20]. The incommensurate phase of Quartz should then be ferroelectric while neither the $\alpha$ nor the $\beta$ phase are. We have then performed experiments on this modulated phase at high temperature, under stress and under the application of an external electric field. 
has been used in order to performed experiments on the triple- $q$ to single- $q$ phase transition of the inc. phase of Quartz. This original stage is described by Caillard et al. in this journal. It only permits to stretch the sample $(\sigma<0)$ and includes a heating resistor. For these experiments the quartz single crystal is cut perpendicular to the $c$ axis into $8 \mathrm{~mm} \times 2 \mathrm{~mm} \times 0,2 \mathrm{~mm}$ thin plates. Two holes are drilled at the extremity to allow for stress application. Samples are then thinned using ion milling until a central hole is achieved. They are then covered with a thin layer of amorphous carbon to avoid electrostatic charging. Radiation damage was minimized by observing the samples at temperatures close to the $\alpha / \beta$ transition (around $850 \mathrm{~K}$ ). Stress is applied at high temperature when the sample is in the 3-q inc. phase but it cannot be quantitatively measured. In agreement with Coujou et al. results [21], we have studied the two higher stressed regions perpendicular to the direction of stress on either side of the central hole.

The experiments about the ferroelectric properties of the incommensurate phase of quartz have been performed using the sample holder presented in $\S 2.3 .1$ with a CM20 Philips microscope. The Quartz samples have been prepared in the same way as described for $\mathrm{BaTiO}_{3}$ compounds.

\subsection{RESUlts.}

4.3.1 Triple- $q$ phase. - Figure 10 depicts the incommensurate phase observed by electron microscopy without any applied stress nor electric field. The micrograph is obtained by using the 110 reflection in the $\left(a^{*}, b^{*}\right)$ plane surrounded by six satellites. The contrasts observed on such micrograph are consistent with a triple- $q$ incommensurate phase and their interpretation has already been discussed in [22]. Here we only report the main results: because of a thermal gradient at the surface sample, a large domain of existence of the inc. phase can be observed between the $\alpha$ phase (left side of the micrograph) and the $\beta$ phase (right side). The triangular dark/light contrast patterns may be interpreted as the result from an interference contrast between the main Bragg reflection and the six satellites spots that surround it at $\pm q i$ value $(i=1,2,3)$. As predicted by the theory of Aslanyan et al. these three $q i$ wave vectors are tilted from the $y$ directions by an angle $\varphi$ depending on the temperature. Then the two equivalent values $+\varphi$ and $-\varphi$ induce the presence of " $\pm \varphi$ macrodomains" structure that is clearly visible in figure 10 . These blocks correspond each to the other by a $\left(180^{\circ}+2 \varphi\right)$ rotation around $c$ axis. The different sizes of the equilateral black and white triangles correspond to the different values of the wave vectors depending on temperature. All the characteristics of this 3-q phase deduced from such electron micrographs are consistent with the results obtained from X-ray, neutron or $\gamma$ diffraction [15-17].

4.3.2 Triple- $q$ phase / single- $q$ phase transition under stress. - Figure 11 displays an electron micrograph observed on a quartz sample under uniaxial stress. It has been achieved using the (110) diffraction spot in the [001] zone axis. As in figure 10, a large domain of the incommensurate phase is visible. Stress direction is reported in the figure and slightly deviates from the $x$ direction. On the right side of the micrograph fringes parallel to the $x$ direction can be observed. They exhibit a periodicity of $20 \mathrm{~nm}$ and correspond to the strip phase with a wave vector parallel to the [100] direction. The contrasts at the center of the figure are quite different from the regular equilateral triangle images observed on the $3-q$ phase in figure 9 . Nevertheless the $120^{\circ}$ angle between the three directions of the modulation indicates the triple- $q$ state. Moreover the presence of the two " $\pm \varphi$ macrodomains" that can be seen in the center of this micrograph proves the triple$q$ state of the incommensurate phase. Indeed these domains are only allowed on the triple- $q$ state when it is not on 1- $q$ state. To account for the contrasts observed in the center of figure 11, changes occurring in the wave vectors have been envisaged. We have used a model proposed by Vallade et al. [23] about the evolution of the wave vector star in the small stress limits. Calculated images using six satellites at $+q i,-q i$ displaced following their model are presented in [24] and agree quite well with our experimental results. Another important feature of this micrograph 


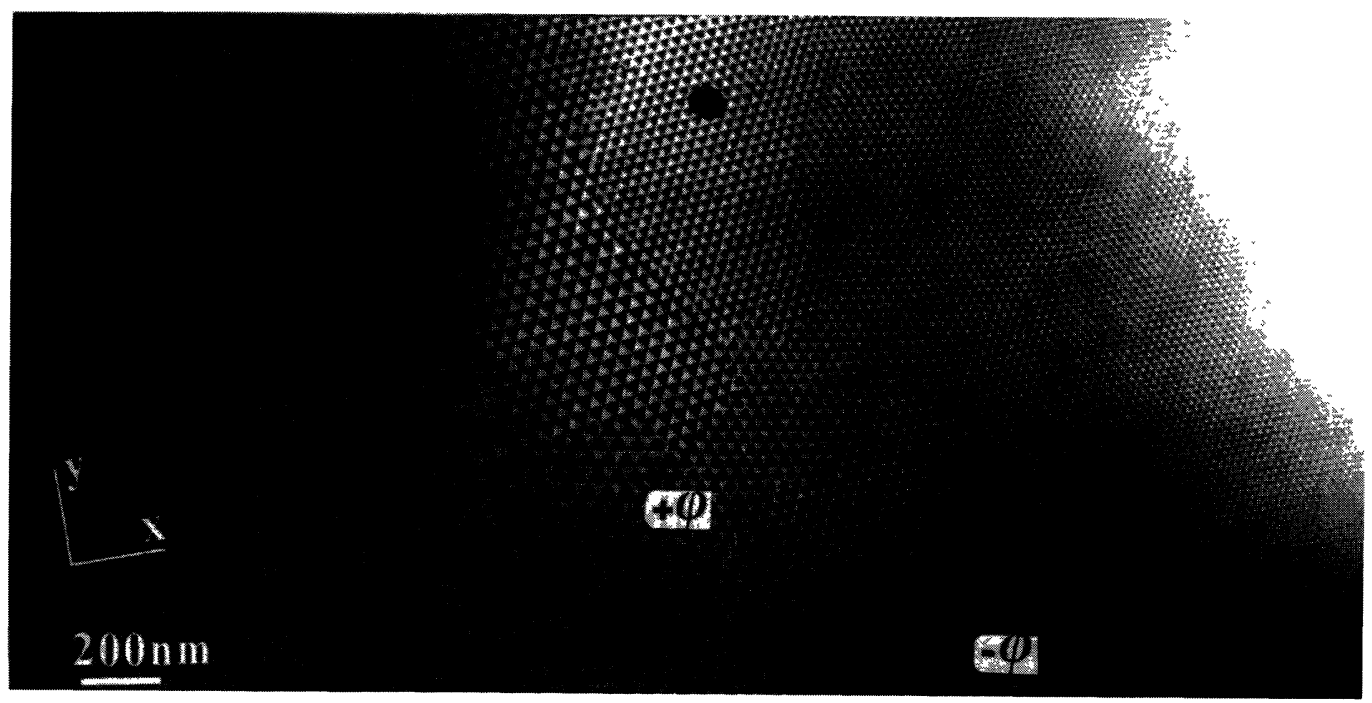

Fig. 10. - Dark field image of the incommensurate phase of Quartz obtained selecting the 110 reflection and its six satellites.

can be observed on the elongated triangle-like structure on the left side of the micrograph in figure 11. Such contrasts can be fitted considering an interference phenomenon between the main reflection and two satellites tilted by an angle $\pm \varphi$ from the $y$ direction. We then assume that this intermediate phase is a double- $q$ one with wave vectors taken from the two different $\pm \varphi$ macrodomains of the triple- $q$ phase without stress. With increasing the uniaxial stress the two wave vectors are approaching each other (the $\varphi$ angle decreases) until they merge to achieve the single- $q$ modulation.

4.3.3 Evidence of the ferroelectric properties of the incommensurate phase of quartz. - The micrograph in figure 12 is a dark field image 110 of the incommensurate phase poled under an external electric field of $4 \mathrm{kV} / \mathrm{cm}$ parallel to the $c$ axis. As in figure 10 domains are clearly observed but are now more numerous: $\pm \varphi$ macrodomains still appear and are labelled $+\mathrm{B}+$ and $-\mathrm{B}-$. Moreover two more kinds of block appear. They are labelled $+\mathrm{B}-$ and $-\mathrm{B}+$ and respectively correspond to $+\mathrm{B}+$ and $-\mathrm{B}-$ by a two fold rotation around $\mathrm{c}$. Some block boundaries are pointed out by arrows. Theoretical assumptions of Janovec and Saint Gregoire [25, 26] forecast the existence of these four types of blocks. Without electric field $+B+$ and $-B-$ are the stable blocks which present antiparallel polarization along $\mathrm{c}$ as they are observed figure 10 while $+\mathrm{B}-$ and $-\mathrm{B}+$ are unstable. With the application of an positive external E. field along $z .+\mathrm{B}+$ stay stable $+\mathrm{B}-$ and $-\mathrm{B}+$ become metastable and $-\mathrm{B}$ - unstable. The micrograph in figure 12 confirms the existence of the four different blocks it agrees with their assumptions about the polarization of the different blocks and evidences the ferroelectric properties of the incommensurate phase of Quartz.

\section{Conclusion.}

We have shown that in situ TEM experiments which is relevant for plasticity and irradiation studies is also efficient for phase transition studies. We have illustrated it and presented various TEM 


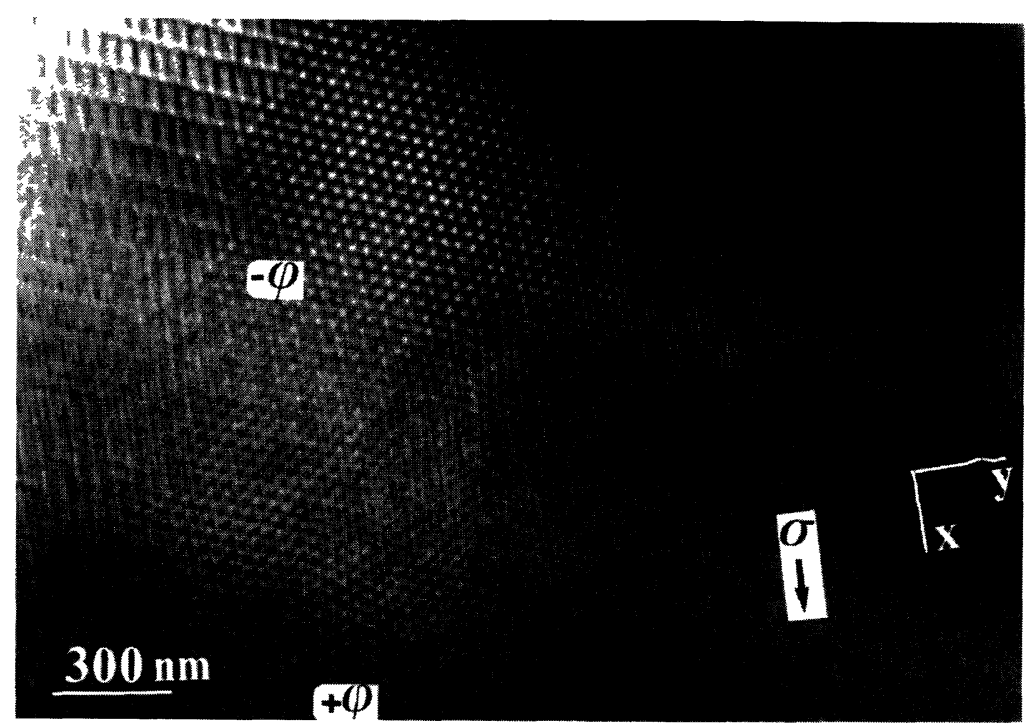

Fig. 11. - (110) dark field image of the stressed incommensurate phase of Quartz. An uniaxial stress applied along $x$ induces a triple- $q$ to single- $q$ phase transition.

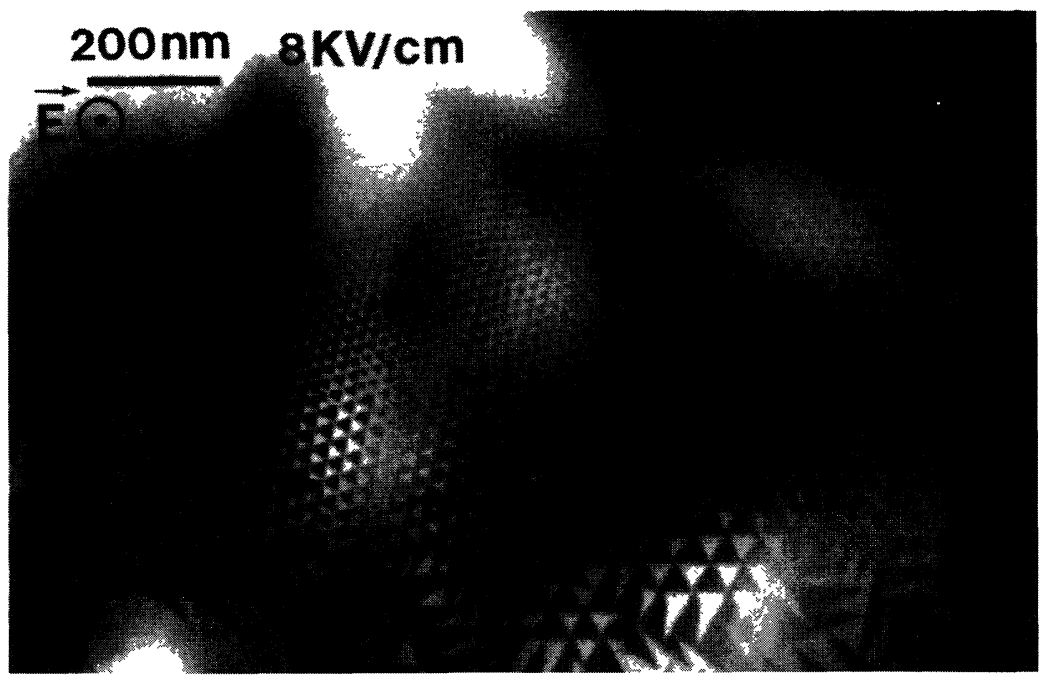

\section{Incommensurate phase of Quartz observea unaer} an external electric field. (110) Dark field image.

Fig. 12. - (110) dark field image of the incommensurate phase of Quartz under an external electric field parallel to the $z$ direction. 
results performed on ferroic (ferromagnetic and ferroelectric/ferroelastic) domain switches using original stages. Phase transitions associated to diffusion processes can also be investigated by TEM using an environmental cell. We have studied the kinetics of the phase transition in ( $\mathrm{Ln}) \mathrm{Ba}_{2} \mathrm{Cu}_{3} \mathrm{O}_{6+x}$ compound and pointed out the effect of the pressure on the $\mathrm{T}-\mathrm{O}$ transition temperature using an experimental device available on the $3 \mathrm{MeV} \mathrm{TEM}$. Incommensurate phase can also be efficiently studied by TEM experiments. We have presented original results performed on the incommensurate phase of Quartz. The use of an heating straining stage has permitted us to study the triple- $q$ to single- $q$ phase transition. Other in situ TEM experiments under an external E. field have been carried out and have evidenced the ferroelectric properties of the incommensurate phase of Quartz.

\section{Acknowledgements.}

The authors wish to thank P. Sopena from the CEMES-LOE/ CNRS for his help in TEM sample preparations, J. Bilotte and C. Jouret for 3 MV TEM experiments.

\section{References}

[1] Newnham R.E. and Cross L.E., Mat. Res. Bull. 9 (1974) 927.

[2] TAYLOR R.A., J. Magnetism Magnetic Mater. 31-34 (1983) 999.

[3] Degauque J., Bouchara D., Fagot M., Bras J., Redoules J.P., Chomel P.H. and Astie B., I.E.E.E. Trans. Magnetics. 26, ${ }^{\circ} 5$ (1990).

[4] Degauque J. and Astie B., Phys. Stat. Sol. (a) 74 (1982) 201.

[5] BORNAREL J., LAJZEROWICZ and LEGRAND Ferroelectrics, 7 (1974) 313-314.

[6] SNOECK E., NORMAND L., ThOREl A. and RouCAU C., submitted to "Phase Transitions".

[7] Locatelli D., Martinez J.P., Balladore J.L. and Jouffrey B., 6th Int. Conf. HVEM, Anvers 4 (1980) 74.

[8] Hodeau J.L., Chaillout C., Capponi J.J. and Marezio M., Solid State Com. 64, n ${ }^{\circ} 11$ (1987) 1349.

[9] Van tendello G., ZandBergen H.W. and AmelinCKS S., Solid State Com. 63, n ${ }^{\circ} 5$ (1987) 389.

[10] Millet P., Enjalbert R., Galy J., Faulmann C., Cassoux P., Rakoto H. and Askenazy S., C.R. Acad. Sci. Paris, 306 Série II (1988) 407.

[11] Baules P., Millet P., Casanove M.J., Snoeck E. and Roucau C., J. Micr. Spectr. Electr. 14 (1989) 305-314.

[12] Van tandelou G., Van landuyt J. and Amelinckx S., Phys. Stat. Sol. (a) 33 (1976) 723.

[13] Van landuyt J., Van tandeloo G., Amelinckx S. and Walker M.B., Phys. Rev. B 31 (1985) 2986.

[14] SNoeck E., Roucau C. and Saint-Gregoire P., J. Phys. 47 (1986) 2041-2053.

[15] Kato N. and Gouhara K., Phys. Rev. B 34, n 3 (1986) 2001-2003.

[16] Gouhara K. and Kato N., J. Phys. Soc. Jpn 53 (1985) 1869.

[17] BASTIE P. and Dolino G., Phys. Rev. B 31, n 5 (1985) 2857-2961.

[18] ASlanyan T.A. and LeVANyuK A.P., Solid State Comm. 31 (1979) 547.

[19] Dolino G., Bastie P., Berge B., Vallade M., Regnault L.P. and Zeyen C.M.E., Europhys. Lett. 3 (5) (1987) 601-609.

[20] Walker M.B., Phys. Rev. B 28 (1983) 6407.

[21] Coujou A., Lours PH., Roy N.A., CaIllard D. and Clement N., Acta metallurgica materialia 32, $n^{\circ} 5$ (1990) 825-837.

[22] SnOeCK E., Roucau C. and MutKa H., J. Phys. 50 (1989) 937-948.

[23] Vallade M. and Petit M., Phys. Stat. Sol. (A) 111 (1989) 155.

[24] SNOeck E. and Roucau C., Phys. Rev. B 45, n 12 (1992) 720-724.

[25] Saint gregoire P., Janovec V., Snoeck E., Roucau C. and Zikmund Z., Ferroelectrics, 125 (1992) 202-214.

[26] SNOECK E., JANOVEC V., SAINT GREgoire and RouCAu C. in preparation. 